\title{
Extension of Transonic Flow Computational Concepts in the Analysis of Cavitated Bearings
}

D. Vijayaraghavan

Lewis Research Center

Cleveland, Ohio

T.G. Keith, Jr.

University of Toledo

Toledo, Ohio

and

D.E. Brewe

Propulsion Directorate

U.S. Army Aviation Systems Command

Lewis Research Center

Cleveland, Ohio

Prepared for the

Joint Tribology Conference

cosponsored by the STLE and ASME

Toronto, Canada, October 7-10, 1990

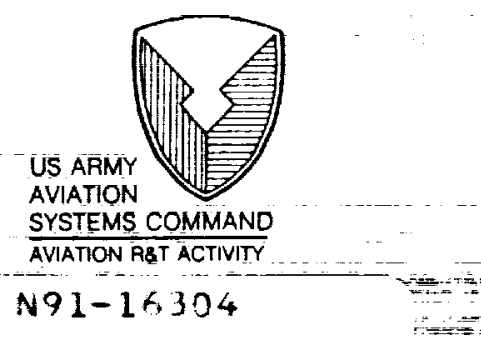




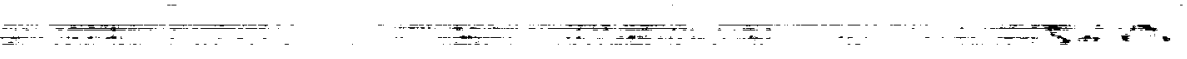

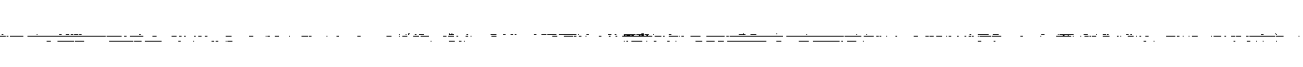

$-\cdots$ 


\title{
Extension of Transonic Flow Computational Concepts in the Analysis of Cavitated Bearings
}

\author{
D. Vijayaraghavan* \\ National Aeronautics and Space Administration \\ Lewis Research Center \\ Cleveland, Ohio 44135 \\ T.G. Keith, Jr. \\ Department of Mechanical Engineering \\ University of Toledo \\ Toledo, Ohio 43606 \\ and \\ D.E. Brewe \\ Propulsion Directorate \\ U.S. Army Aviation Systems Command \\ Lewis Rescarch Center \\ Cleveland, Ohio 44135
}

\begin{abstract}
In this paper, an analogy between the mathematical modeling of transonic potential flow and the flow in a cavitating bearing is described. Based on the similarities, characteristics of the cavitated region and jump conditions across the film reformation and rupture fronts are developed using the method of weak solutions. The mathematical analogy is extended by utilizing a few computational concepts of transonic flow to numerically model the cavitating bearing. Methods of shock fitting and shock capturing are discussed. Various procedures used in transonic flow computations are adapted to bearing cavitation applications, for example, type differencing, grid transformation, an approximate factorization technique, and Newton's iteration method. These concepts have proved to be successful and have vastly improved the efficiency of numerical modeling of cavitated bearings.
\end{abstract}

\section{Introduction}

Cavitation in fluid film bearings, though recognized as early as 1886 when Reynolds introduced the theory of lubrication, is still a subject which draws intense debate as to the nature and mechanism of the phenomena. Various theories and conditions for cavitation have been put forward. However, only the collective works of Jakobsson and Floberg (1957) and Olsson (1965), now known as JFO theory, have provided insight into the subject, which is both consistent with mass conservation and the physics of the problem. When the boundary conditions developed in JFO theory are applied to the Reynolds equation, the extent of cavitated regions and the performance of bearings can be predicted more precisely than by any existing method. This theory has yielded results which are in good agreement with experimental data.

The subject of gas dynamics gained immense research interest around the turn of this century. This effort helped promote the development of supersonic aircrafts and large thrust rocket nozzles. Application of gas dynamics principles include turbine flows, gas lasers, aerodynamic windows, missile aerodynamics, jet engines and the flow around a body entering the atmosphere (Emanuel, 1986). Mach number $M$, a nondimensional parameter, which is the ratio of the flow speed to the local speed of sound, is the indicative index as to whether the flow is subsonic $(M<1)$, sonic $(M=1)$, or supersonic $(M>1)$. It is also a

*National Research Council-NASA Research Associate at Lewis Research Center. 
measure of the compressibility of the flow. In a convergingdiverging duct, the flow can range from subsonic to supersonic or the reverse. The converging section is called a nozzle when the flow is subsonic and is accelerating; it is called a diffuser when the flow is supersonic and is decelerating. The diverging section is called a diffuser when the flow is subsonic and is decelerating and is called a nozzle when the flow is supersonic and is accelerating. When subsonic and supersonic flow regimes exist, the flow is called transonic. It is also possible for an internal flow to be totally subsonic or supersonic through the nozzle. However, for a transonic flow to exist, a duct with a throat is essential.

Prior to 1965 , computational methods were rarely used in aerodynamic analysis and importance was placed on expensive and time consuming wind tunncl experiments. With the emergence of powerful computers, computational aerodynamics has greatly advanced to the extent that the flow pattern past entire aircraft in different flight regimes can be predicted (Jameson, 1987). Such rapid growth in computational techniques can be attributed to its direct application in the design of aircraft and space vehicles. In addition, there was little recourse aside from expensive experimentation, due to the nonlinear nature of the goveming equations which made them intractable to analytical modeling. Developments in computational methods applied in the lubrication area have been comparatively slow to evolve. In fact, to date, the numerical algorithms developed by Elrod (1981) and Kumar and Booker (1989) are the only effective numerical tools available in the analysis of cavitation in bearings.

Transonic flow theory and the theory of lubrication are two distinctly different fields as far as the physical phenomena are concerned. While transonic flow theory deals with compressible fluid flow near sonic velocity, classical lubrication theory is generally concerned with the flow of a highly viscous incompressible fluid with a Reynolds number that is very small. However, due to the existence of subsonic and supersonic flow regimes in a convergingdiverging nozzle and the existence of full film and cavitated regions in a bearing with a converging- diverging wedge, there exists a striking resemblance in the mathematical modeling of these two problems. Such an analogy can substantially benefit either field by suitably incorporating the advancements from one field to the other.

In this paper, a mathematical formulation for a cavitated bearing is derived and compared with that of transonic potential flow. An analogy between these formulations are developed. The analogy is utilized to employ the method of characteristics and the method of weak solutions from transonic flow theory to determine the characteristics of a cavitated region and the jump conditions that apply across both film reformation and rupture fronts. The method of determining the location of film reformation using shock fitting and shock reformation techniques are discussed. A brief discussion of several techniques that are widely used in current transonic flow computation is provided. These techniques have already been suitably modified and incorporated into the analysis of cavitation in bearings.

\section{Mathematical Modeling}

\section{Cavitated Bearing}

The conservation of mass flow, within the clearance between the stationary and moving surfaces of a bearing can be written, by lumping across the film thickness, as

$$
\frac{\partial \rho \mathbf{h}}{\partial \mathbf{t}}+\nabla \cdot \mathbf{m}=0
$$

In the converging wedge of the bearing, the film thickness diminishes and the pressure is developed. In this region, the mass flux $m_{\mathrm{f}}$ can be represented by

$$
m_{f}=\frac{U}{2} \rho h-\frac{\rho h^{3}}{12 \mu} \nabla p
$$

The first term on the right side is the mass flow due to shear (Couette flow) and the second term is the flow due to pressure gradient (Poiscuille flow). Somewhere in the diverging wedge of the bearing, the film ruptures and a cavitation region is formed which continues until the film is reformed again. In this cavitated region, the pressure remains essentially constant at the cavitation pressure and the mass flows across this region due only to shear along the film striations. In the cavitated region, the film occupies only a portion of the volume, the remaining portion being filled by air, gas, or vapor. Mass flow in this region is given by

$$
\mathbf{m}_{\mathbf{c}}=\frac{\mathrm{U}}{2} \rho_{\mathrm{c}} \mathbf{h} \theta
$$

where $\theta$ is the partial film content in the cavitated region. Although the film consists of incompressible fluid, if it is assumed that the density of the film varies due to the applied pressure, then the variable $\theta$ can be provided with a dual interpretation

$$
\theta=\left\{\begin{array}{l}
\rho / \rho_{c}, \text { in the full film region where } \theta \geq 1 \\
V_{\mathrm{f}} / \mathrm{V}_{\mathrm{l}}, \text { in the cavitated region where } \theta<1
\end{array}\right.
$$

where $V_{f}$ and $V_{t}$ are the volume occupied by the film and the total volume, respectively. In the full film region, the variation of density will be governed by the bulk modulus of the liquid, that is,

$$
\beta=\rho \frac{\partial p}{\partial \rho}=\theta \frac{\partial p}{\partial \theta}
$$


Equation (4) also enables one to represent the pressure in terms of the density (or $\theta$ ), and in essence, acts as the equation of state of the lubricant.

Mass flow through the entire bearing can then be written as

$$
\mathbf{m}=\frac{\mathrm{U}}{2} \rho_{c} \mathbf{h} \theta-g \frac{\rho_{c} \beta \mathbf{h}^{3}}{12 \mu} \nabla \theta
$$

where $g$ is a switch function, which is introduced to remove the flow due to pressure gradient within the cavitated region and is defined by

$$
\mathrm{g}=\left\{\begin{array}{l}
1 \text { when } \theta \geq 1 \\
0 \text { when } \theta<1
\end{array}\right.
$$

For a finite bearing, the flow due to shear occurs only in the circumferential direction while the flow due to pressure gradient is present in both the circumferential and axial directions. Hence, the two dimensional form of equation (1) can be written as

$$
\begin{array}{r}
\frac{\partial \rho_{c} \theta \mathbf{h}}{\partial t}+\frac{\partial}{\partial \mathbf{x}}\left(\frac{\rho_{c} \mathbf{h U} \theta}{2}-\frac{\rho_{c} \beta \mathbf{h}^{3}}{12 \mu} \mathrm{g} \frac{\partial \theta}{\partial \mathbf{x}}\right) \\
+\frac{\partial}{\partial z}\left(-\frac{\rho_{c} \beta \mathbf{h}^{3}}{12 \mu} \mathrm{g} \frac{\partial \theta}{\partial z}\right)=0
\end{array}
$$

Equation (6), which can be used to describe the mass flow through the entire bearing, was developed by Elrod and Adams (1974) and termed a 'universal equation'. In the full film region, equation (6) may be written as

$$
\frac{\partial \mathbf{E}}{\partial \mathbf{t}}+\frac{\mathbf{U}}{2} \frac{\partial \mathbf{E}}{\partial \mathbf{x}}=\frac{\partial}{\partial \mathbf{x}}\left(\mathbf{K} \frac{\partial \theta}{\partial \mathbf{x}}\right)+\frac{\partial}{\partial \mathbf{z}}\left(\mathbf{K} \frac{\partial \theta}{\partial \mathbf{z}}\right)
$$

where $\mathbf{E}=\theta \mathbf{h}$ and $K=-\beta h^{3} \mathrm{~g} / 12 \mu$. Equation (7) is an elliptic partial differential equation. The form of equation (6) in the cavitated region is given by

$$
\frac{\partial E}{\partial t}+\frac{U}{2} \frac{\partial E}{\partial x}=0
$$

and is a hyperbolic partial differential equation. This may be easily demonstrated by differentiating with respect to $t$ to get

$$
\frac{\partial^{2} E}{\partial t^{2}}+\frac{U}{2} \frac{\partial^{2} E}{\partial x \partial t}=\frac{\partial^{2} E}{\partial t^{2}}-\frac{U^{2}}{4} \frac{\partial^{2} E}{\partial x^{2}}=0
$$

Equation (9) is a canonical form of the wave equation. The characteristic form of this equation has two real roots, $\pm \mathrm{U} / 2$.

In the full film region, pressure increases to a maximum value and then gradually decreases until the pressure and its derivative simultaneously vanish, at a location where the film ruptures. The air/gas/vapor strips in the cavitated region begin with a pointed shape. When the film reforms, depending upon the upstream conditions, the reformation occurs abruptly. This is because the cavitated region is not able to signal the impending conditions to the upstream flow. Figure 1 represents typical profiles of pressure and fractional film content for a submerged journal bearing, at a particular axial location. The abrupt changes in $\theta$ and the pressure gradient at the reformation boundary and the gradual changes at the rupture boundary can be clearly

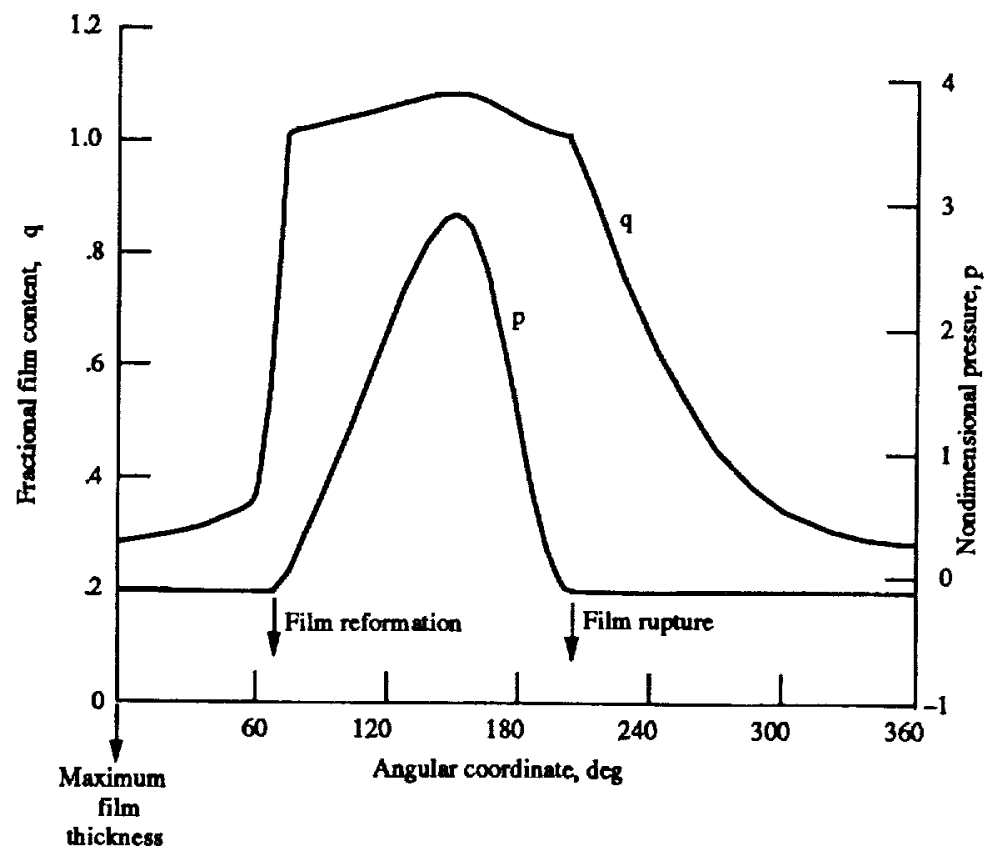

Figure 1.-Typical pressure and fractional film content distributions in a journal bearing. 
seen. When the effects of cavitation are not formally considered, it is usually assumed that the film ruptures at the minimum film thickness and reforms at the maximum film thickness. When the cavitation boundaries are determined, it is found that the film extends slightly beyond the minimum film thickness into the diverging portion of the bearing and, depending upon the lubricant supply conditions, the film reformation can occur at or around the feed groove.

\section{Transonic Flow}

The flow of a compressible fluid in thermodynamic equilibrium is governed by the Navier-Stokes equations. For a two-dimensional flow these equations can be written in vector form as

$$
\frac{\partial w}{\partial t}+\frac{\partial f}{\partial x}+\frac{\partial g}{\partial y}=\frac{\partial R}{\partial x}+\frac{\partial S}{\partial y}
$$

where $w$ is the vector of dependent variables: density, Cartesian velocity components, and total energy; $\mathbf{f}$ and $\mathbf{g}$ are the convective flux vectors; and $R$ and $S$ are the viscous flux vectors. Because the full Navier-Stokes equations are quite complex, approximations are generally made. One such simplification consists of assuming no viscous dissipation and that flow is irrotational. Consequently, equation (10) can be written in a quasi-linear form in terms of the velocity potential $\phi$.

$$
\left(1-\frac{u^{2}}{c^{2}}\right) \phi_{x x}-2 \frac{u v}{c^{2}} \phi_{x y}+\left(1-\frac{v^{2}}{c^{2}}\right) \phi_{x y}=0
$$

It should be noticed that, in a subsonic flow regime $(\mathrm{u}, \mathrm{v}<\mathrm{c})$, the coefficients of the second order terms will be positive and, in a supersonic flow regime $(u, v>c)$, the coefficients become negative. This variation results in the equation being of the elliptic type with two imaginary roots in subsonic regions and of the hyperbolic type with two real roots in supersonic regions.

When a subsonic flow slows down, it does so gradually. On the other hand, a supersonic flow, which can also decelerate gradually, normally slows down abruptly. Because the fluid in a supersonic flow is unable to signal the upstream flow of any flow or geometric changes. This is a typical characteristic of phenomena governed by hyperbolic-type equations. The abrupt changes lead to discontinuities in the flow which are known as shock waves and are a distinct feature of a supersonic flow in establishing the overall nature of the flow field. Of course, an internal flow can also emerge as supersonic without the presence of any shock if the outlet conditions permit. Since viscous effects are neglected in the potential equation, for internal flow, sonic conditions occur at the throat of the duct. For real fluids, the sonic line extends slightly downstream of the throat into the diverging portion of the duct.

Computational techniques for potential flow have been extensively developed, since, extremely inexpensive computation is achieved with this formulation. Moreover, shock capturing and convèrgence accelcration techniques developed for potential how have been found to be transferable to more complex models using Euler equations.

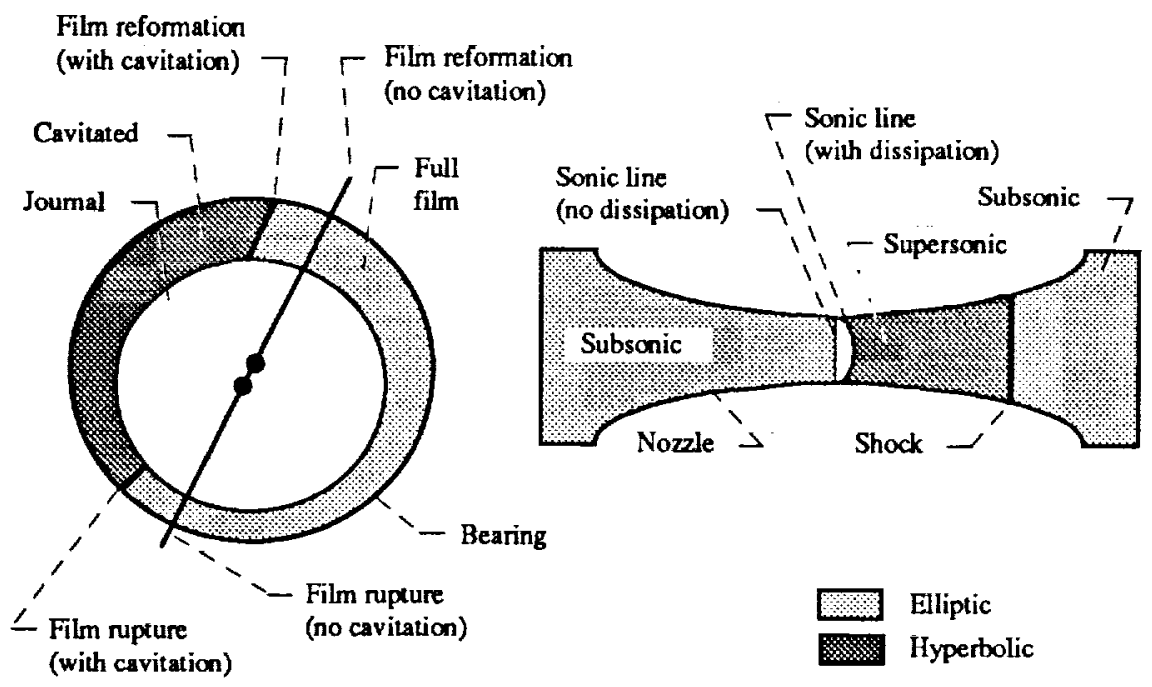

Figure 2.-Transonic flow and cavitated bearing. 


\section{Analogy}

The similarities between internal transonic flow and cavitated bearing modeling are evident from the previous two sections. Figure 2 illustrates these similarities. Both phenomena are governed by similar mathematical formulations in the different regions and both have an embedded hyperbolic region within the elliptic region. The subsonic portion of the flow $(M<1)$ is analogous to the full film region $(\theta>1)$. The sonic line $(M=1)$ is analogous to film rupture $(\theta=1)$. The supersonic portion of the flow $(M>1)$ is analogous to the full film region $(\theta<1)$. Also, a shock and film reformation have similar characteristics. It should be recognized that the Mach number and the inverse of fractional film content have similarities. The sonic line/ the film rupture locations are strongly influenced by the geometry of the flow, while the shock wave/film reformation locations are due to the upstream conditions. The flow can also be fully elliptic or hyperbolic in both cases, although a completely cavitated bearing has no physical significance. Similar to compressible flows involving shocks, determination of the film reformation boundary is a difficult task.

Although, it is seen that transonic flow and flow in a cavitated bearing have similarities, it should also be noted that they also differ in several respects. The essential differences between these two models are the following:

(1) In transonic flow, the type of the equation is changed due to the change in the sign of the coefficient of the second order terms; in the case of a cavitated bearing, the second order terms are totally lost in the hyperbolic region resulting in the reduction of the order of the goveming equation. This sometimes causes oscillations at the boundary locations.

(2) The entire flow is compressible in a transonic flow; but, in a cavitated bearing, the full film region flow although really incompressible is taken to be compressible and the cavitated region flow is of the compressible type.

(3) The unknowns in an irrotational transonic flow are density and the potential function which are dependent on each other. On the other hand, for a cavitated bearing the only unknown is density (or $\theta$ ).

(4) Flow in both Cartesian directions can occur in transonic flow, although the resultant velocity can be made to align with one of the coordinate axes by Jameson's rotated difference scheme (1974). In bearings, generally the only motion is a direct result of the journal rotation and the velocity vector is normally taken to coincide with a coordinate axis.
Having pointed out the basic analogy between the two models, extension of method of characteristics to determine the path of the disturbances in the cavitated region and the method of weak solutions to determine the jump conditions across the discontinuities can be developed. Also, determination of the location of film reformation using shock fitting and shock capturing methods will be discussed. Since, our intention is to develop corresponding expressions for a cavitated bearing based on the analogy, the development details for transonic flow are not presented in detail, here. Interested readers are refered to, for example, Anderson et al. (1984).

\section{Method of Characteristics}

Hyperbolic equations have certain lines (or surfaces) which indicate the zones of influence and zones of dependence. The information about the flow is signalled along these lines which are called characteristics. This property is used to determine the value of the variable at a particular location in a hyperbolic region from the known value of the variable at a downstream location. This method of solving hyperbolic equations is the Method of Characteristics (MOC).

Supersonic flow. - Assuming the free stream is aligned with the $x$ axis, equation (11) can be written as

$$
\left(1-M_{\infty}^{2}\right) \phi_{x x}+\phi_{y y}=0
$$

where $\mathbf{M}_{\infty}$ is the freestream Mach number. In order to determine the characteristic direction, equation (12) is written in terms of the Cartesian velocity components along an arbitrary smooth curve $C$, and the determinant of the coefficients is set to zero along the curve. This will result in the differential equations for the characteristics. For this case,

$$
\left.\begin{array}{l}
\frac{d y}{d x}= \pm \frac{1}{B} \\
B^{2}=\left(M_{\infty}^{2}-1\right)
\end{array}\right\}
$$

For a constant $B$, the equations describing the characteristics are obtained by integrating equation (13). This results in the following form:

$$
\left.\begin{array}{l}
\xi=\mathbf{x}-\mathbf{B y} \\
\eta=x+B y
\end{array}\right\}
$$


where $\xi$ and $\eta$ are coordinates along the characteristic line.

Cavitated region. - Within the cavitated region, the governing hyperbolic equation is written as

$$
\frac{\partial \mathbf{E}}{\partial t}+\frac{U}{2} \frac{\partial \mathbf{E}}{\partial \mathbf{x}}=0
$$

Along a curve $x=x(t)$ in the $x-t$ plane, $E=E(x)$. For $a$ particular curve $x=x_{c}(t)$, let $d E=0$.

Thus,

$$
d E=\frac{\partial E}{\partial t} d t+\frac{\partial E}{\partial x} d x=0
$$

Using equation (15), results in

$$
-\frac{U}{2} \frac{\partial E}{\partial x} d t+\frac{\partial E}{\partial x} d x_{c}=\frac{\partial E}{\partial x}\left(\frac{\partial x_{c}}{\partial t}-\frac{U}{2}\right) d t=0
$$

Therefore,

$$
\left.\begin{array}{c}
\frac{\partial \mathbf{x}_{\mathrm{c}}}{\partial \mathrm{t}}=\frac{\mathrm{U}}{2} \\
\frac{\partial \mathbf{E}}{\partial \mathrm{t}}=0
\end{array}\right\}
$$

The solution to the first of equation (18) determines the equation of the characteristics and the second one reveals the parameter that is constant along the characteristics. They are

$$
\left.\begin{array}{c}
\xi=x_{c}-\frac{U}{2} t \\
E=\theta h=\text { constant }
\end{array}\right\}
$$

Since $h$ is known, the value of $\theta$ at any point in the cavitated region can be obtained from a point with a known value of $\theta$ by tracing backwards along the characteristic line. Olsson (1965) discussed this characteristics approach in his treatise on dynamically loaded bearings.

\section{Method of Weak Solutions}

A genuine solution of a hyperbolic differential equation is one in which the dependent variable is continuous but discontinuities in its derivatives may occur. Alternately, a weak solution is genuine except along a surface across which the dependent variable is discontinuous. Only the scalar or vector form of first order and hyperbolic second order partial differential equations possess weak solutions. Since the dependent variable is not continuous across the discontinuity, an integral formulation is used.
This eliminates the need for the solution to be differentiable across the discontinuity. The mathematical theory of weak solutions for hyperbolic equations is a relatively recent development and may be utilized to determine the jump conditions across a discontinuity in a flow.

Transonic flow. - Although the steady state formulations exhibit elliptic and hyperbolic type equations at different parts of the flow field, addition of an unsteady term results in hyperbolic type of equation. Consider a one-dimensional, scalar, nonlinear, hyperbolic partial differential equation

$$
\frac{\partial u}{\partial t}+\frac{\partial F}{\partial x}=0
$$

where $\mathrm{u}$ and $\mathrm{F}(\mathrm{u})$ are unknown variables. This can be rewritten as

$$
\frac{\partial \mathbf{u}}{\partial \mathbf{t}}+\mathbf{A} \frac{\partial \mathbf{u}}{\partial \mathbf{x}}=0
$$

where $\mathbf{A}=\mathbf{A}(\mathbf{u})=\mathbf{d F} / \mathbf{d u}$ is called the Jacobian. Now, if $w(x, t)$ is an arbitrary test function which is continuous and has a continuous first derivative but vanishes on the boundary and outside of an arbitrary domain $D$ in the $(x, t)$ plane, then

$$
\iint_{D}\left(\frac{\partial u}{\partial t}+\frac{\partial F}{\partial x}\right) w(x, t) d x d t=0
$$

When both $u$ and $F$ are continuous and have continuous first derivatives, it can be shown that

$$
\iint_{D}\left(u w_{t}+F w_{x}\right) d x d t=0
$$

Functions $u(x, t)$ which satisfy equation (23) for all test functions $w$ are called weak solutions of equation (21). If the domain $D$ contains a moving curve $\tau(x, t)$, across which $\mathrm{u}$ is discontinuous as shown in figure 3 , equation (23) may be integrated by parts, utilizing equation (22), to get

$$
\int_{\tau}\left([u] \cos \alpha_{1}+[F] \cos \alpha_{2}\right) d s=0
$$

where [ ] denotes a jump across the discontinuity and $\cos \alpha_{1}$ and $\cos \alpha_{2}$ are the direction cosines normal to the discontinuity $\tau(\mathrm{x}, \mathrm{t})$. Since the integrand must vanish for all $w$, the condition for $u$ to be a weak solution of equation (21) will therefore be

$$
[\mathrm{u}] \cos \alpha_{1}+[\mathbf{F}] \cos \alpha_{2}=0
$$

Equation (25) determines the jump in the value of $\mathrm{u}$ across the discontinuity. This analysis is also valid for a system of equations in which case, $u$ and $F$ are vectors. The jump conditions, 


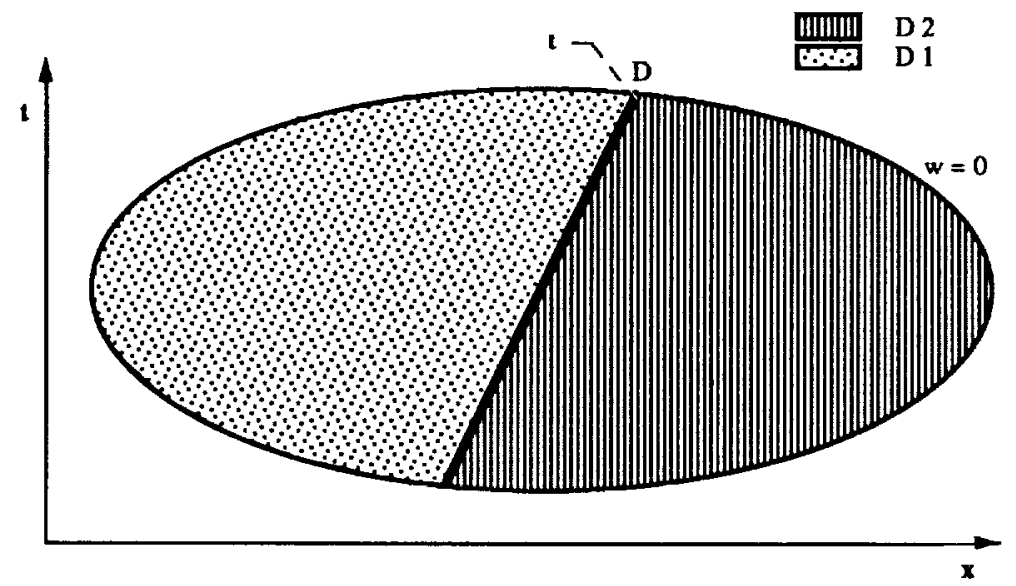

Figure 3.-An arbitrary domain with a discontinuity.

$$
[u] \frac{d x}{d t}=[F]
$$

where $d x / d t$ is the velocity of the discontiuity, are popularly known as the Rankine-Hugoniot equations. For example, if $\mathbf{u}$ and $\mathbf{F}$ are vectors defined as

$$
\begin{gathered}
u=\left[\rho, \rho u, \rho e+\rho \frac{u^{2}}{2}\right]^{T} \\
F=\left[\rho u, \rho u^{2}+p, u\left(\rho e+\rho \frac{u^{2}}{2}\right)+p\right]^{T}
\end{gathered}
$$

then the Rankine-Hugoniot equations can be written as

$$
\begin{gathered}
\mathbf{U}_{d}[\rho]=[\rho \mathbf{u}] \\
\mathbf{U}_{d}[\rho \mathbf{u}]=\left[\rho \mathbf{u}^{2}+p\right] \\
\mathbf{U}_{d}\left[\rho \mathbf{e}+\rho \mathbf{u}^{2} / 2\right]=\left[\mathbf{u}\left(\rho \mathbf{e}+\left(\rho \mathbf{u}^{2} / 2\right)+p\right)\right]
\end{gathered}
$$

where $U_{d}$ is the velocity of discontinuity and e is the internal energy. The first two equations are called mechanical jump conditions.

Cavitated bearing. - Consider the one-dimensional version of equation (6), which can be written as

$$
\frac{\partial \mathbf{E}}{\partial \mathbf{t}}+\frac{\partial \mathbf{m}}{\partial \mathbf{x}}=0
$$

where $m$ is the mass flux as defined by equation (5). Equation (28) can be written in a form similar to that of equation (21), that is,

$$
\frac{\partial \mathbf{E}}{\partial \mathbf{t}}+\mathbf{A} \frac{\partial \mathbf{E}}{\partial \mathbf{x}}=0
$$

where $\mathbf{A}=\mathbf{A}(\mathbf{E})=\mathbf{d m} / \mathbf{d E}$. To proceed in the same manner as that used for the transonic flow illustration, it can be shown that for $E$ to be a weak solution of equation (28), the jump condition to be satisfied at any discontinuity is

$$
\text { [E] } \cos \alpha_{1}+[\mathrm{m}] \cos \alpha_{2}=0
$$

At any time $t_{1}$, the velocity of propagation of the discontinuity is determined by the tangent of the curve as shown in figure 4 . The direction cosines are

$$
\cos \alpha_{1}=\frac{1}{\left(1+\frac{d t}{d x}\right)_{t=t_{1}}^{\frac{1}{2}}} \text { and } \cos \alpha_{2}=-\frac{\frac{d t}{d x}}{\left(1+\frac{d t}{d x}\right)_{t=t_{1}}^{\frac{1}{2}}}
$$

therefore,

$$
\frac{\cos \alpha_{1}}{\cos \alpha_{2}}=-\frac{d x}{d t}
$$

Hence, equation (30) can be written in terms of the primitive variables as

$$
\begin{aligned}
& {\left[(\theta h)_{L}-(\theta h)_{R}\right] \frac{d x}{d t}-\left\{\frac{U}{2}\left[(\theta h)_{L}-(\theta h)_{R}\right]\right.} \\
& \left.-\frac{\beta}{12 \mu}\left[\left(h^{3} g(\theta) \frac{\partial \theta}{\partial x}\right)_{L}-\left(h^{3} g(\theta) \frac{\partial \theta}{\partial x}\right)_{R}\right]\right\}=0
\end{aligned}
$$

where $L$ and $R$ are the conditions at the left and right sides of the discontinuity, respectively. In other words,

$$
[\theta \mathbf{h}] \frac{\mathbf{d x}}{\mathrm{dt}}=[\mathbf{m}]
$$

is an equivalent Rankine-Hugoniot equation. 


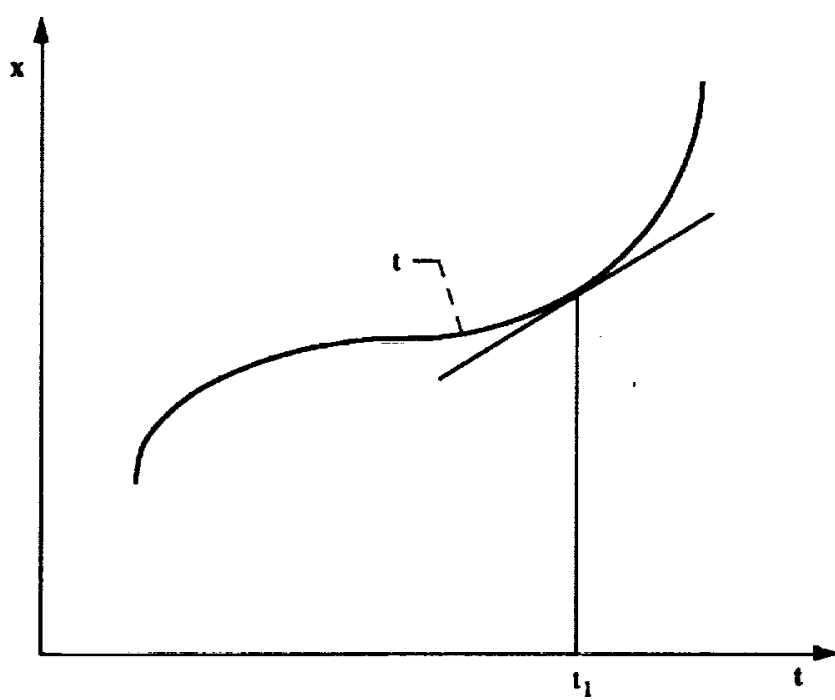

Figure 4.-Path of a discontinuity.

First consider the discontinuity due to film reformation. If it is assumed that the film thickness variation across the discontinuity is negligible and that $\theta_{R}=1$ since full film is formed at the right side of the discontinuity, equation (32) for these conditions can be written as

$$
\left(\theta_{L}-1\right) \frac{d x}{d t}-\left\{\frac{U}{2}\left(\theta_{L}-1\right)-\frac{\beta}{12 \mu}\left[-\left(h^{2} \frac{\partial \theta}{\partial x}\right)_{R}\right]\right\}=0
$$

If the reformation front is not moving with respect to time (analogous to a stationary shock), then the conditions for film reformation will be

$$
\frac{\mathbf{U}}{2}\left(\theta_{L}-1\right)=\frac{\beta}{12 \mu}\left[-\left(h^{2} \frac{\partial \theta}{\partial \mathbf{x}}\right)_{\mathbf{R}}\right]
$$

Since $(\partial \theta / \partial x) \geq 0$, obviously, $\theta_{L} \leq 1$.

On the other hand, if the reformation front is in motion, equation (34) can be written as follows

$$
\left(\frac{U}{2}-\frac{d x}{d t}\right)\left(\theta_{L}-1\right)=-\frac{\beta}{12 \mu}\left(h^{2} \frac{\partial \theta}{\partial x}\right)_{R}
$$

Since $(\partial \theta / \partial x) \geq 0$, the right side of equation (36) is less than or equal to zero. Also, since $\theta_{L} \leq 1$, there can be two conditions

\section{(i) $\mathrm{dx} / \mathrm{dt} \geq \mathrm{U} / 2$}

For this condition, the left side of equation (36) is greater than or equal to zero. Hence, the only condition that will satisfy the equality is that both sides must be zero, that is, $(\partial \theta / \partial x)_{R}=0$ and $\theta_{L}=1$. This is the classical Reynolds boundary condition which is generally applied to determine film rupture. (ii) $\mathrm{dx} / \mathrm{dt}<\mathrm{U} / 2$

Now, the left side of equation (36) is also less than or equal to zero. Hence, $q_{\mathrm{L}}$ cannot be uniquely determined from equation (36); it must be obtained using the characteristics. Equation (36) is treated as a differential equation of the following form to determine the velocity of the front:

$$
\frac{d x}{d t}=\frac{\dot{U}}{2} \cdot \frac{\beta}{12 \mu} \frac{1}{\left(1-\theta_{L}\right)}\left(h^{2} \frac{\partial \theta}{\partial x}\right)_{R}
$$

Although in transonic flow, the sonic line is not treated as a discontinuity; in the case of a dynamically loaded, cavitated bearing, there could be a discontinuity of $\theta$ at the film rupture, when $\mathrm{dx} / \mathrm{dt}>\mathrm{U} / 2$ and there will be a jump in its gradient. The jump conditions for this case can be developed in the same manner as previously described. The resulting expressions will be

(i) $d x / d t \leq U / 2(\partial \theta / \partial x)_{L}=0$ and $\theta_{R}-1$

(ii) $d x / d t>U / 2 \quad \frac{d x}{d t}=\frac{U}{2}+\frac{\beta}{12 \mu} \frac{1}{\left(\theta_{R}-1\right)}\left(h^{2} \frac{\partial \theta}{\partial x}\right)_{L}$

These jump conditions are exactly the same as those determined by Olsson (1965). However, in that reference the conditions were derived using a mass balance across a fluid volume containing the discontinuity.

\section{Computational Treatment of Discontinuity}

Transonic flow. - The numerical computation of supersonic flow is complicated due to the presence of shock waves, across which the dependent variables and 
their derivatives may be discontinuous. Two types of numerical techniques have been developed to analyze such flow fields and are known as shock fitting and shock capturing techniques.

Shock fitting technique. - This technique attempts to locate any discontinuities and treats them as boundaries between the regions of the flow field where regular solutions are applicable. Shock fitting is achieved by satisfying the Rankine-Hugoniot equations across the discontinuities while simultaneously ensuring that the solution on the downstream side of any shock is compatible with the rest of the flow field. The movement of the shock wave is obtained as a part of the solution. The flow field downstream of each shock can be determined from freestream conditions. If the upstream conditions, initial shock slope and velocity are known, the shock acceleration and post shock pressure can be determined by combining RankineHugoniot equations with the compatible equation. This technique is most convenient for governing equations written in nonconservative form.

Several approaches have been devised to fit shocks (Moretti, 1974). The flow field is either partitioned by aligning any shock waves with grid lines or the discontinuities are treated explicitly, but not as boundaries, in the computation.

Shock capturing technique. - Unlike the shock fitting technique, with this method, the discontinuities are predicted as a part of the solution without the requirement of any special treatment. By casting Euler equations in conservation-law form, the weak solutions and jump conditions are built in. The conservative form of governing equations and the discretization automatically allow prediction of the shock wave speed and the strength (Lax, 1954).

Because of the simplicity in approach, this technique is most popular in the computation of flows with shocks. This technique also has several variants which include flux splitting and split coefficient matrix methods. Shock waves predicted with this technique can be smeared over several grid spaces and the application of surface boundary conditions can be difficult. Due to the wavelike nature of hyperbolic equations, boundary errors are propagated into the flow field which results in instability in the computation. In general, shock capturing techniques are applied to predict internal shocks while boundary shocks are fitted.

Cavitated bearing. - Similar to the previously described approaches for transonic flow, discontinuity fitting and capturing techniques can be applied to problems with cavitating regions. Determining the film reformation boundary is more difficult than determining the film rupture boundary due to sudden changes in the flow variables across the front.

If the initial location and slope of the boundaries are not known, they can be determined by employing a trial and error method (the discontinuity fitting method). The location of the boundaries can be assumed and the flow field on either side of the discontinuity can be determined. The equivalent Rankine-Hugoniot equation can then be applied across the boundary to verify the assumption. If the initial location and slope are known, then the governing equations coupled with the equivalent Rankine-Hugoniot equation can be solved to determine the new velocity and the new locations of the discontinuities.

The algorithm introduced by Elrod (1981) is essentially a discontinuity capturing technique. By combining the goverming equations for the full film and cavitated regions and conserving mass flow through the entire bearing, the 'universal equation' is cast intoa conservationlaw form. Hence, the discontinuities can be predicted as a part of the solution. This method is simple to implement and does not require any knowledge about the location of the discontinuities. The boundaries are predicted very effectively.

\section{Concepts from Transonic Flow Computation}

The authors have utilized a few transonic flow computational concepts in the analysis of cavitated bearings. The following is a brief discussion of this work.

\section{Computational Algorithm}

When the potential flow equation was used in transonic flow computation, difficulty was encountered due to the reversal of the velocity vector in the supersonic flow regime. Murman and Cole (1971), in a landmark paper, demonstrated a simple way to obtain a meaningful solution by proposing the use of central differencing in the subsonic region (elliptic) and one sided upwind differencing in the supersonic region (hyperbolic). Jameson (1975) created a type differencing scheme by introducing an artificial viscosity term into the governing equation. This enables automatic switching of the form of differencing as required within different regions of the flow. Also, with the explicit addition of an artificial viscosity term, the conservation form of the equation was preserved.

In the analysis of cavitation in bearings, Elrod (1981) modified the originally proposed algorithm by Elrod and 
Adams (1974), by incorporating the idea of changing the form of differencing in the full film and cavitated regions. However, this effect was achieved by 'trial and error' and the algorithm was empirically developed. A type differencing scheme was developed by Vijayaraghavan and Keith (1989), by introducing an artificial viscosity term (much like Jameson's) into the governing equation which in turn permitted the algorithm to be mathematically derived. In addition, with this modification, the discretization was also performed in conservative form. The predictions using this modified algorithm were found to compare well with the predictions using the Elrod algorithm and with experimental data. Hence, the modified cavitation algorithm, with this firmer base, was thought to offer greater potential for further improvement.

\section{Grid Control}

Numerical grid generation is a fairly common tool to model arbitrarily shaped regions in computational fluid dynamics. This is basically a procedure to distribute in an orderly manner the grid points in the physical field in such a way that efficient communication between the points and all the physical phenomena on the entire continuous field is represented with sufficient accuracy (Thompson, 1984). Also, the region in the immediate vicinity of solid surfaces are dominant in determining the character of the flow due the large gradients prevailing in this region. Accurate prediction of flow variables in this region is important since the final values of the variables strongly depends on this boundary prediction. When such high gradient regions are not known a priori, a dynamically adaptive grid system can be an effective tool. This is an active area in grid generation research. By dynamically readjusting the grid distribution as the solution proceeds, high resolution solutions can be obtained with fewer grid points.

In the case of a cavitated bearing, film rupture and reformation locations are not known ápriori. Also, accurate prediction of the pressure distribution is the primary requirement in the full film region, since, all the performance parameters depend upon the pressure profile. Hence, closely placed grid points around the cavitation boundaries and more grid points in the high pressure gradient regions should lead to a more accurate prediction of the pressure profile. In the case of a bearing with a misaligned journal, the maximum pressure location in the axial direction is shifted towards the edge of the bearing and to correctly predict the pressure distribution in this region, closely spaced grid points are required. With a uniformly fine grid arrangement, this will result in a large number of grid points located within regions where such a small grid spacing does not necessarily provide any significant improvement in the accuracy. With a grid adaption technique all these effects can be achieved by moving the grid points and selectively locating them in such a way that accurate solutions can be obtained with fewer grid points. The concept of grid generation/transformation and grid adaption techniques have been incorporated into the modified cavitation algorithm by Vijayaraghavan and Keith (1990(a)).

The grid adaption procedure can be tailored to the problem being solved. However, the procedure envisaged was to perform initial computations which locate the film rupture and reformation fronts, todistribute closely spaced grid around them, and then to rearrange the grid in the full film region according to the pressure gradient. In the case of a misaligned journal, when the degree of misalignment is large, grid adaption in the axial direction is applied to cluster the grid around the maximum pressure location (Vijayaraghavan and Keith, 1990(b)). By aligning the grid with discontinuities, the flow field is divided into zones of full film and cavitated regions. This enables the jump conditions to be applied effectively. In addition, in the case of a two-dimensional time asymptotic solution, by aligning the discontinuity along one coordinate direction, according to the equivalent Rankine-Hugoniot equation, the mass flow along the other coordinate direction is continuous across the discontinuity. This method of grid adaption combines features of both shock fitting and shock capturing techniques.

The predicted performance of the bearings using the adaptive grid and conventional orthogonal grid arrangements were found to be comparable. The results obtained thus far demonstrate the usefulness of these techniques in the analysis of bearing problems. The transformation of the governing equation and numerical differencing in a nonorthogonal coordinate system could be confidently applied, primarily due to the mathematical base provided in the modified algorithm.

\section{Solution Procedure}

In the case of transonic flow, for steady problems, the converged solution obtained by using relaxation methods generally requires a very large number of iterations due to the slow convergence rate. The two most effective solution acceleration techniques for rapid convergence in the transonic flow computations are approximate factorization of the difference operators and the use of multiple grids (Jameson,1987). For genuine unsteady transonic flow problems, Newton iteration techniques can be applied to the governing equation, at every time step, to obtain time accurate solutions (Shankar et al, 1985). 
In the analysis of cavitated bearings, relaxation methods are found to also require a large number of iterations to obtain a converged steady state solution. Woods and Brewe (1989), in the analysis of a dynamically loaded, submerged joumal bearing, incorporated a multigrid technique into the Elrod algorithm and obtained considerable savings of computer time. In an unsteady analysis of cavitated bearings, particularly dynamically loaded bearings, time accurate solutions are very important. In such cases, the accuracy of the solution can be improved by adding a Newton iteration technique. The Newton iteration scheme and approximate factorization techniques were developed by Vijayaraghavan and Keith (1990(c)) for the modified algorithm. The approximate factorization technique was found to be robust and efficient. The unique advantage of these techniques is that with the same unsteady formulation, both time accurate unsteady results and fast convergent asymptotic steady state solutions can be obtained in less computer time than by using a steady state formulation.

\section{Conclusion}

Recognition of the mathematical similarities between internal transonic flow and cavitated bearing flow is an important and useful concept. To the best of authors' knowledge, such similarities have not been pointed out before. The analogy has been exploited to determine the characteristics within the cavitated region and the jump conditions across any discontinuity in the flow field. By virtue of similarities between the two flows, advanced concepts of transonic flow computations can be incorporated into numerical predictions of cavitation in bearings. Determination of the reformation boundary using both shock fitting and shock capturing methods are discussed. With the conservative formulation of the governing 'universal equation', the shock capturing method is very effective and simple to implement. The concepts of transonic flow computation have been developed and successfully incorporated in three important areas, namely, algorithm development, grid arrangement and control, and efficient solution computation. The results obtained are encouraging and it is believed that many more such extensions may be possible which will result in improved numerical prediction of cavitation in bearings.

\section{References}

Anderson, D.A., Tannehill, J.C., and Pletcher, R.H., 1984, Computational Fluid Mechanics and Heat Transfer, Hemisphere Publishing Corp., Washington, D.C.

Elrod, H.G., 1981, "A Cavitation Algorithm," Journal of Lubrication Technology, Vol. 103, No. 3, pp. 350-354.
Elrod, H.G., and Adams, M.L., 1974, "A Computer Program for Cavitation and Starvation Problems," Cavitation and Related Phenomena in Lubrication, D. Dowson, M. Godet, and C.M. Taylor, eds., Mechanical Engineering Publications, New York, pp. 37-41.

Emanuel, G, 1986, Gas dynamics: Theory and Applications, AIAA Education Series, AIAA New York.

Jakobsson, B., and Floberg, L., 1957, "The Finite Journal Bearing, Considering Vaporization," Transactions of Chalmers University of Technology, Guthenberg, Sweden, No. 190.

Jameson, A., 1987 "Success and Challenges in Computational Aerodynamics," 8th Computational Fluid Dynamics Conference, AIAA, New York, pp. 1-35.

Jameson, A., 1974, "Iterative Solution of Transonic Flows Over Airfoils and Wings, Including Flows at Mach 1," Communications on Pure and Applied Mathematics, Vol. 27, No. 3, pp. 283-309.

Jameson, A., 1975, "Transonic Potential Flow Calculations using Conservation Form," Proceedings of 2 nd Computational Fluid Dynamics Conference, AIAA, New York, pp. 148-161.

Kumar, A., and Booker, J.F., 1989, “A Finite Element Cavitation Algorithm," ASME Paper 89-Trib-59.

Lax, P.D., 1954, "Weak Solutions of Nonlinear Hyperbolic equations and Their Numerical Computation," Communications on Pure and Applied Mathematics, Vol. 7, No. 1, pp. 159-193.

Moretti, G., 1974, "On the Matter of Shock Fitting," Proceedings of the Fourth International Conference on Numerical Methods in Fluid Dynamics, Lecture Notes in Physics, Vol. 35, R.D. Richtmyer, ed., SpringerVerlag, New York, pp. 287-292.

Murman, E.M., and Cole, J.D., 1971, "Calculation of Plane Steady Transonic Flows," AIAA Journal, Vol. 9, pp. 114-121.

Olsson, K.O., 1965, "Cavitation in Dynamically Loaded Bearings," Transactions of Chalmers University of Technology, Guthenberg, Sweden, No. 308.

Shankar, V., Ide, H., Gorski, J., and Osher, S., 1985, "A Fast Time-Accurate Unsteady Full Potential Scheme," AIAA Journal, Vol. 25, No. 2, pp. 230-238.

Thompson, J.F., 1984, "Grid Generation Techniques in Computational Fluid Dynamics," AIAA Journal, Vol. 22, No. 11, pp. 1505-1523.

Vijayaraghavan, D., and Keith, T.G., Jr., 1989, “Development and Evaluation of a Cavitation Algorithm," STLE Tribology Transactions, Vol. 32, No. 2, pp. 225-233. Vijayaraghavan, D., and Keith, T.G., Jr., 1990(a), "Grid Transformation and Adaption Techniques Applied in the Analysis of Cavitated Joumal Bearings," Journal of Tribology, Vol. 112, No. 1, pp. 52-59. 
Vijayaraghavan, D., and Keith, T.G., Jr., 1990(b), "Analysis of a Finite Grooved Misaligned Journal Bearing Considering Cavitation and Starvation Effects," Journal of Tribology, Vol. 112, No. 1, pp. 60-67.

Vijayaraghavan, D., and Keith, T.G., Jr., 1990(c), "An Efficient, Robust, and Time Accurate Numerical Scheme Applied to a Cavitation Algorithm," Journal of Tribology, Vol. 112, No. 1, pp. 44-51.
Woods, C.M., and Brewe, D.E., 1988, "The Solution of the Elrod Algorithm for a Dynamically Loaded Journal Bearing using Multigrid Techniques," Journal of Tribology, Vo. 111, No. 2, pp. 302-308. 


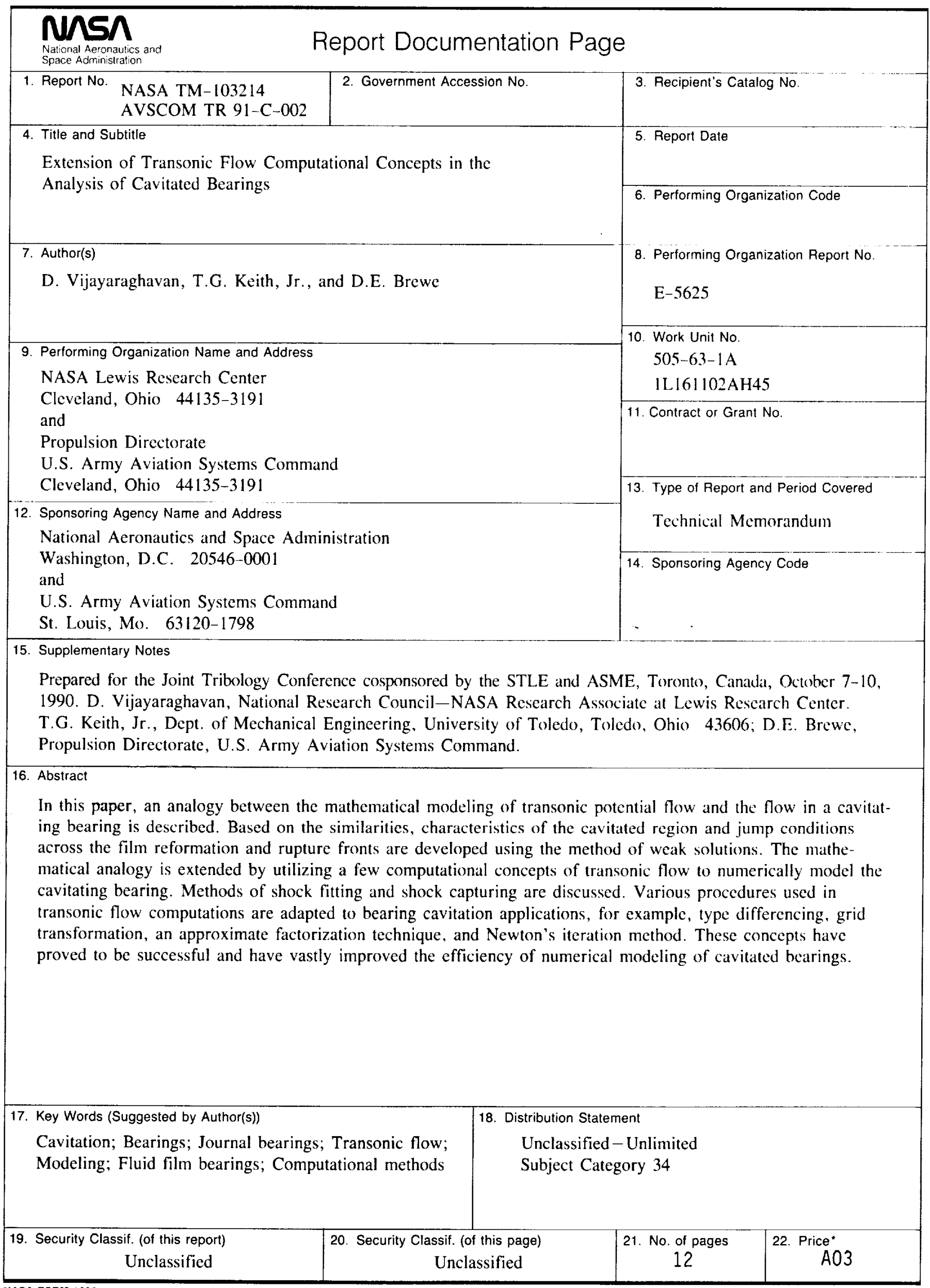


